\title{
Evaluating the Influence of Individualized Nursing Care on Chinese Postpartum Women
}

\author{
Linping Huang ${ }^{1}$, Xinke Huang ${ }^{2, *}$, Wenchan Feng ${ }^{1}$ \\ ${ }^{1}$ Baby-friendly Zone, The First Affiliated Hospital of Jinan University, Guangzhou, China \\ ${ }^{2}$ The Department of Obstetrics and Gynecology, The First Affiliated Hospital of Jinan University, Guangzhou, China \\ Email address: \\ 52848826@qq.com (Liinping Huang),929901838@qq.com (Xinke Huang), 13802549408@163.com (Wenchan Feng) \\ ${ }^{*}$ Corresponding author
}

\section{To cite this article:}

Linping Huang, Xinke Huang, Wenchan Feng. Evaluating the Influence of Individualized Nursing Care on Chinese Postpartum Women. Journal of Gynecology and Obstetrics. Vol. 8, No. 2, 2020, pp. 45-47. doi: 10.11648/j.jgo.20200802.13

Received: March 17, 2020; Accepted: April 3, 2020; Published: April 23, 2020

\begin{abstract}
Objective: To evaluate the influence of individualized nursing care on Chinese postpartum women. Methods: We invited 182 participants to join our study, who are childbirth in the First Affiliated Hospital of Jinan University, their childbirth time is from April 2016 to October 2019. They were randomly assigned to control group $(n=91)$ and intervention group $(n=91)$, the two group receive different nursing services, such as individualized nursing care and traditional nursing services. We use Self-Rating Anxiety Scale (SAS), Self-rating depression scale (SDS) and other questionnaires to collect the data from participants, the data include patient comfort status, patient satisfaction status, anxiety status and depression status. Result: In comfort research, the comfort status of most patient is 0 level in control group and intervention group $(n=44 \& n=56)$. On the other hand, the control group had more 1 level assessment in this result $(\mathrm{n}=22)$. In satisfaction report, the performance of individualized nursing care is better than traditional nursing services in satisfaction [86 (94.5\%) vs 77 (84.6\%)]. In SDS and SAS research, The intervention group had greater improvement than that of control group in the SAS as the result of intervention group lowered scores even more. Conclusion: the individualized nursing care have great improvement to improve hospital satisfaction and mental health of patients. But the individualized nursing care has little effect on improving patient comfort.
\end{abstract}

Keywords: Individualized Nursing Care, Postpartum Women, Mental Health

\section{Introduction}

Individualized nursing care is a planned care in nursing services, its aim is meet the particular needs which each patient has across the disease continuum, even if these needs might change over time, regardless the routine applied to other patients with a similar disease [1]. In individualized nursing care process, the patients and their families constitute integral components and prerequisites for quality healthcare were decide the decision making and delivery processes, because individualized nursing care is patient- and family-centered [2]. In order to patients receive the best possible method for treatment and care, healthcare providers who provide individualized nursing care have to develop an individualized healthcare plan, through co-operation and exchange of opinions with the patient and the specialists that treat them, regarding the best possible method for treatment and care
[3-5]. In this method, the patient's needs may be identified and plans to satisfy them may be articulated.

In China, postpartum women are increasingly vulnerable to suffer from poor mental health, because they had a consequence of raising stress and less accessible social support [6]. Base on some reports, $31.4 \%$ of perinatal women from low- and middleincome countries were living with a depressive disorder and $17 \%$ were living with major depressive disorder, the data shown that the prevalence of poor mental health varied greatly among studies $[7,8]$. Other studies further reported discrepancies in mental health between the prenatal and postpartum periods. Such as the risk of depression are raised in the postpartum period when compared to the prenatal $[9,10]$. Aim of this study is assess the influence of individualized nursing care on Chinese postpartum women. 


\section{Methods}

\subsection{Participants Enrollment and Survey Methods}

182 patients was invited to join our study, who are childbirth in the First Affiliated Hospital of Jinan University. The participants' childbirth time is from April 2016 to October 2019. All participants were randomly assigned to two groups, that included a control group $(\mathrm{n}=91)$ and a intervention group $(n=91)$. The intervention group and control group receive different nursing services after childbirth, that the control group participants receive traditional nursing services, which is build from past hospital standard. On the another hand, the intervention group patients receive individualized nursing care after childbirth. We collected the information from all participants, the information included patient comfort status, patient satisfaction status, anxiety status and depression status. In addition, we collected data using the following questionnaires: Self-Rating Anxiety Scale (SAS), Self-rating depression scale (SDS) and other questionnaires [11, 12].

The individualized nursing care is provide personalized care services on the basis of patient's habits, medical records and personal preferences. Our researchers deliberately analyzes patients' personalized information so that we can provide appropriate personalized care services to corresponding patients.

Their inclusion criteria were: (1) The patient successfully complete the childbirth; (2) Patients volunteered to participate in follow-up; (3) They did not suffer from severe postpartum complications. Their withdraw criteria were: (1) The patient was hospitalized at another medical facility after childbirth; (2) Poor mental health.

\subsection{Statistical Analysis}

Our data analyzer performed the statistical analysis by SPSS 22.0. The $\mathrm{P}$ value, t-test and chi-square test were associated with collection result were analyzed. Besides, the mean standard deviation for statistical description.

\section{Result}

In comfort level research, most participants had qualified comfort services in the nursing process, this research shown total comfort status of participants (Table 1). In 0 level result, the comfort status of most patient is 0 level in control group and intervention group $(n=44 \& n=56)$. On the other hand, the control group had more 1 level assessment in this result $(\mathrm{n}=22)$.

Table 1. Comfort level [n (\%)].

\begin{tabular}{lllll}
\hline Projects & Qualified comfort level & 0 level & 1 level & 2 level \\
\hline Intervention group $(n=91)$ & $85(93.4 \%)$ & $56^{*}$ & 16 & 13 \\
Control group $(n=91)$ & $79(86.8 \%)$ & 44 & 22 & 6 \\
\hline
\end{tabular}

$* \mathrm{P}<0.05$.

Base on Table 2, the satisfaction result indicated that the intervention group patient think that they had better satisfaction to individualized nursing care, the performance of individualized nursing care is better than traditional nursing services in satisfaction [86 (94.5\%) vs $77(84.6 \%)]$. In total, most satisfaction assessment is very good level, the very good level assessment of intervention group is more than that of control group.

Table 2. Patient satisfaction [n (\%)].

\begin{tabular}{llllll}
\hline Projects & Satisfaction rate & Very good & good & Pass & Bad \\
\hline Intervention group $(\mathrm{n}=91)$ & $86(94.5 \%)^{*}$ & $68^{*}$ & 9 & 7 & $7^{*}$ \\
Control group $(\mathrm{n}=91)$ & $77(84.6 \%)$ & 51 & 11 & 15 & 14 \\
\hline
\end{tabular}

$* \mathrm{P}<0.05$.

This result shown the change of anxiety status and depression status in control group and intervention group (Table 3). We ask the participants finish the questionnaires when the nursing services beginning, and ask they complete the the questionnaires again after the nursing services. The intervention group had greater improvement than that of control group in the SAS as the result of intervention group lowered scores even more.

Table 3. The score of anxiety and depression from SDS and SAS (Mean \pm SD).

\begin{tabular}{llll}
\hline Projects & & SAS & SDS \\
\hline \multirow{2}{*}{ Intervention group (n=91) } & BN & $57.5 \pm 6.8$ & $57.7 \pm 6.9$ \\
& FN & $49.2 \pm 6.1$ & $52.7 \pm 5.5$ \\
Control group (n=91) & BN & $61.4 \pm 8.5$ & $60.7 \pm 5.7$ \\
\hline
\end{tabular}

$\mathrm{BN}=$ before the nursing.

$\mathrm{FN}=$ after the nursing.

\section{Discussion}

The postpartum period is a physiological and a critical transition time for a woman and her newborn. Some of the care related problems in the postpartum period are the causes of maternal and infant mortality [13]. Base on some reports, infant and maternal mortality rates are on the rise in developing country, such as China and India. In TWO report of 2015 , maternal mortality rates was 239 per 100,000 live births in developing countries in 2015 , the infant mortality rate was 32 deaths per 1000 live births in 2015 [14, 15]. So barriers such as poverty, lack of information, inadequate services, and cultural practices that limit access to quality maternal health services must be identified and addressed at all levels of the health system to prevent maternal and infant deaths [16]. In the 2018 report of the United States, 1 in every 10 women will 
experience a preterm delivery, which is defined as birth occurring before 37 weeks of gestation [17]. But traditional nursing service of hospital is not suitable for all types of maternity, so the individualized nursing care can try to fix this problem in some extent, in spite of its price is expensive. Individualization of care has been defined as both a synonym of patient-centered care and one of its attributes [18]. although there are some studies reporting that organizational factors may influence nurses' ability to individualize care, the detailed relationship between individualized care and the professional practice environment has not been investigated widely.

Base on our research result, the individualized nursing care have great improvement to improve hospital satisfaction and mental health of patients. But the individualized nursing care has little effect on improving patient comfort. In comfort research, research result gap is not great in statistics, intervention group only has a little better performance in some different level. In addition, more intervention group patients report they are satisfied with the individualized nursing care, the number is more than that of control group. In 0 level of satisfaction research, the intervention group assessment is $13.1 \%$ more than the control group assessment. In Self-Rating Anxiety Scale and Self-rating depression scale, the individualized nursing care has stronger influence than that of traditional nursing services. The reduction in anxiety is much greater in the intervention group than in the control group, it also explains why intervention group greater satisfaction assessment. On the other side, the reduction in depression is similar between intervention group and control group. In our study limit, we lack investigation be associate with physiological status, the most collection of our study are psychological aspects. So there is no way to know if the improvement in mental health is due to physical improvements. Moreover, few patient records of past decreased the nursing effect of individualized nursing care.

\section{References}

[1] Charalambous A, Adamakidou T, Cloconi C, Charalambous M, Tsitsi T, Vondrackova L, Buzgova R. The quality of oncology nursing care: a cross sectional survey in three countries in Europe. Eur. J. Oncol. Nurs. 2017; 27: 45-52.

[2] Hughes R. Overview and summary: patient-centered care: challenges and rewards. Online J. Issues Nurs. 2016; 16 (2): 3 9.

[3] Coyle N. Palliative care, hospice care and bioethics - a natural fit. J. Hospice Palliat. Nurs. 2015; 16: 6-12.

[4] Shuman AG, Montas SM, Barnosky AR, Smith LB, Fins JJ,
McCabe MS. Clinical ethics consultation in oncology. J. Oncol. Pract. 2016; 9: 240-245.

[5] Wiegand D, Russo MM. Ethical consideration. In: Dahlin, C. M., Lynch, M. T. (Eds.), Core Curriculum for the Advanced Practice Nurse. Hospice and Palliative Nurses Association, Pittsburgh, PA. 2013: 39-59.

[6] Rahman A, Fisher J, Bower P, Luchters S, Tran T, Yasamy MT, Saxena S, Waheed W. Interventions for common perinatal mental disorders in women in low- and middle-income countries: a systematic review and meta-analysis. Bull. World Health Organ. 2013; 91: 593-601I.

[7] Fellmeth G, Fazel M, Plugge E. Migration and perinatal mental health in women from low- and middle-income countries: a systematic review and meta-analysis. BJOG. 2017; 124: 742752 .

[8] Lindsay M, Burnett E. The use of narcotics and street drugs during pregnancy. Clinical Obstetrics and Gynecology. 2018; 56 (1): 133-141.

[9] Fawcett EJ, Fairbrother N, Cox ML, White IR, Fawcett JM. The prevalence of anxiety disorders during pregnancy and the postpartum period: a multivariate bayesian meta-analysis. J. Clin. Psychiatry. 2019: 80.

[10] Top ED, Cetisli NE, Guclu S, Zengin EB. Paternal depression rates in prenatal and postpartum periods and affecting factors. Arch. Psychiatr. Nurs. 2016: 30: 747-752.

[11] White D, Leach C, Sims R, Atkinson M, Cottrell D. Validation of the Hospital Anxiety and Depression Scale for use with adolescents. Br J Psychiatry 1999; 175: 452-454.

[12] El-Rufaie O, Absood G. Validity study of the Hospital Anxiety and Depression Scale among a group of Saudi patients. Br J Psychiatry 1987; 151: 687-688.

[13] Poorolajal J, Alafchi B, Najafi Vosoogh R, Hamzeh S, \& Ghahramani M. Risk factors for maternal mortality in the west of Iran: A nested case-control study. Epidemiol Health. 2016; 36, e2014028.

[14] Say L, Chou D, Gemmill A, Tuncalp O, Moller AB, Daniels J. Alkema L. Global causes of maternal death: A WHO systematic analysis. The Lancet Global Health. 2016; 2 (6): e323-333.

[15] WHO. Global Health Observatory (GHO) data. Infant mortality. Situation and trends. 2015. Retrieved from http://www.who.int/gho/child_health/mortality/neonatal_infan t_text/en/.

[16] WHO. Maternal mortality. Fact sheet No. 348. 2015. Retrieved from http://www.who.int/mediacentre/factsheets/fs348/en.

[17] Centers for Disease Control and Prevention. National Vital Statistics Reports. 2018; 67 (8): 50.

[18] Brooker D. Person Centred Dementia Care: Making Services Better. Jessica Kingsley, London. 2007. 